\section{IJIE}

Journal homepage: http://penerbit.uthm.edu.my/ojs/index.php/ijie ISSN : 2229-838X e-ISSN : 2600-7916
The International

Journal of

Integrated

Engineering

\title{
Performance Evaluation of DYMO and OLSRv2 Routing Protocols in VANET
}

\author{
Fares Abdulhafidh Dael ${ }^{1}$, Uğur Yavuz ${ }^{1}$, Waheb A. Jabbar ${ }^{2,3 *}$ \\ ${ }^{1}$ Faculty of Economics and Administrative Sciences, Department of Management Information Systems, \\ Üniversite Mahallesi, Atatürk Üniversitesi Kampüsü, Yakutiye/Erzurum, 25030, TURKEY \\ ${ }^{2}$ Faculty of Electrical \& Electronic Engineering Technology, \\ Universiti Malaysia Pahang, 26600 Pekan, Pahang, MALAYSIA \\ ${ }^{3}$ IBM Centre of Excellence, \\ University Malaysia Pahang, 26300 Gambang, Pahang, MALAYSIA \\ *Corresponding Author
}

DOI: https://doi.org/10.30880/ijie.2020.12.01.005

Received 15 October 2018; Accepted 26 December 2018; Available online 31 January 2020

\begin{abstract}
Vehicular Ad hoc Networks (VANET) is one of the emerging mobile ad hoc networking paradigms (MANET), where the self-organizing and infrastructure-less nature of MANET structure. In VANETs, vehicle nodes communicate with each other using wireless links. However, the nodes are highly mobile and the topology of the network changes rapidly. Therefore, the design of routing protocols in VANETs is still a crucial issue. In this paper, we examine two of the most recent routing protocols that proposed for MANETs to select the optimal path between source-destination pairs, namely: Dynamic MANET On-demand (DYMO) and Optimized Link State Routing version 2 (OLSRv2) routing protocols. We evaluated and compared the performance of both protocols based on different parameters under various simulation scenarios. The result has shown that the DYMO protocol has higher through put and packet delivery ratio compare to the OLSRv2 protocol. However, the OLSRv2 protocols has better performance in the terms of average jitter and end to end delay compare to the DYMO protocol based on the paper's scenario. OLSRv2 should be selected if the system concerns about time delay and jitter, otherwise it should be selected DYMO to achieve higher throughput and high packet delivery.
\end{abstract}

Keywords: MANET, VANET, Routing Protocols, DYMO, OLSRv2, QualNet

\section{Introduction}

The recent evolution of wireless technologies offers new perspectives in the field of telecommunications and enables users to communicate in a Device-to-Device (D2D) communication manner regardless of time and place. Mobile AdHoc Network is a set of mobile and/or fixed autonomous devices which are interconnected by a wireless technology, thus, forming a temporary dynamic network with or without the aid of any centralized administration or any fixed support. These networks play an increasingly and important role nowadays because of the variety of applications available on such networks [1][2]. One of the advantages of the multi-hop wireless communication is its capability to extend the connectivity in such way that two nodes [3][4].

Vehicular Ad hoc Network (VANET) is one of the emerging MANETs networking paradigms where the selforganizing and infrastructure-less nature of this paradigm are a plus and not a limitation [2][5]. It is known as application-driven networks that enable information exchange between drivers and concerned authorities for efficient driver assistance and car safety. In VANETs, each vehicle can communicate with other vehicles (V2V) and with roadside (V2R) where wireless communication devices are installed in Road Side Units (RSUs) [6]. The combination of vehicles 
and RSUs forms a dynamic multi-hop ad hoc network where nodes (vehicles) move in an organized manner [7]. Figure 1 shows a typical example of VANET.

The features of VANET and MANET are typically similar in the sense that both of them are self-organization and self-management and do not require to have fixed base station. However, the key difference of VANET is the high speed and tentative mobility of the mobile nodes (vehicles) compared to the nodes in MANET [8]. This fact indicates that the competent design of routing protocol requires improving the MANET architecture to efficiently accommodate the fast mobility of the VANET nodes. This issue has introduced numerous research challenges to the design of a suitable routing protocol [6].

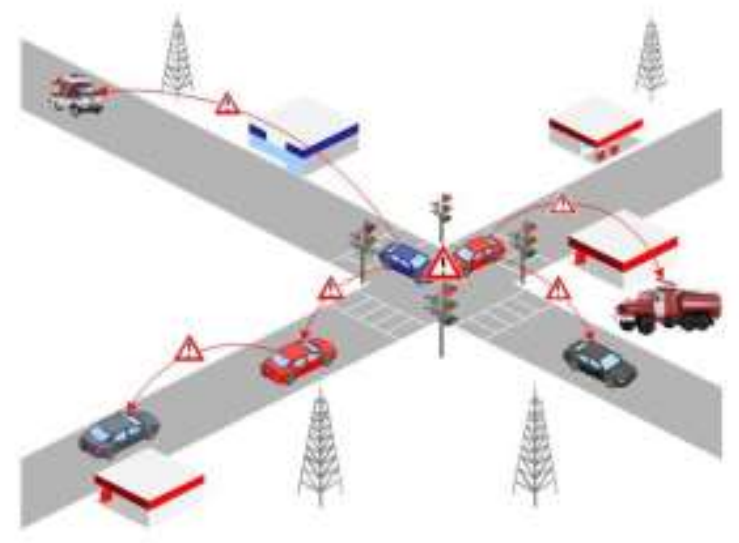

Fig. 1 - VANET communication

The efforts of the Internet Engineering Task Force (IETF) MANET working group are being made to standardize the OLSRv2 [9, 10], a successor to OLSR, and Dynamic MANET On-demand (DYMO) [11, 12], which is currently known as AODVv2 [13], a successor to both AODV and DSR routing protocols. In this paper, we focus on evaluating the performance of these two routing protocols (DYMO and OLSRv2) in VANET environment where the routing is a major networking issue due to the frequent topology changes. General background on these protocols, as well as their concepts and functionalities are provided and described in detail in the next section.

This paper is organized as follows. Section II presents background on routing protocols. In the III related work. Methodology and simulation model are described in Section IV. Section V discusses and compares the simulation results. Finally, conclusion is drawn up in section VI.

\section{Background}

Routing is methodology of moving data from a transmitter to receiver where the data experiences at least one moderate node or hop along the way. But Routing protocol decides the best route between the source and destination to let create network communication. The appropriate route is selected based on routing algorithms.

\subsection{Dynamic Manet On Demand}

DYMO routing protocol is a unicast reactive routing protocol which is intended to be used by mobile nodes in wireless multihop networks and became known as AODVv2 [13]. In this context, Routing Message (Control Packet) is generated only when the node receives a data packet and it does not have any routing information. The most attractive specification defined in the DYMO draft is the internet connectivity [14]. In DYMO protocol, there are two basic operations, namely; Route Discovery and Route Maintenance.

Route Discovery: It is performed when a DYMO router must transmit a packet towards a destination for which it does not have a route as described in Figure 2 [15]. The Route Request (RREQ) routing message is generated for a target node for which it does not have any routing formation. The source node floods the RREQ message to find the target node. During flooding, each intermediate node records a route to the originating node by adding the routing information into this routing table. When the target node receives the RREQ, it responds with a Route Reply (RREP) message which is sent as a unicast message towards the originating node. Each node that receives the RREP records a route to the target node and forwards the RREP to the next hop. When the originating node receives the RREP, routes between the originating node and the target node in both directions are established.

Route Maintenance: It is performed to avoid prematurely expunging routes from the route table, and to avoid dropping packets when an active route breaks. To react to the changes in the network topology, nodes maintain their routes and monitor their links. A Route Error (RERR) message is generated by a node whenever it receives a data packet for a destination to which it has no route in its routing table. This RERR RM notifies other nodes that the current route is broken. Once the source receives the RERR, it re-initiates the route discovery if it still has packets to deliver. HELLO Messages, can be used by nodes to maintain routes to all their neighboring nodes. Sequence numbers are used to avoid routing loops and propagation of stale routing information. 


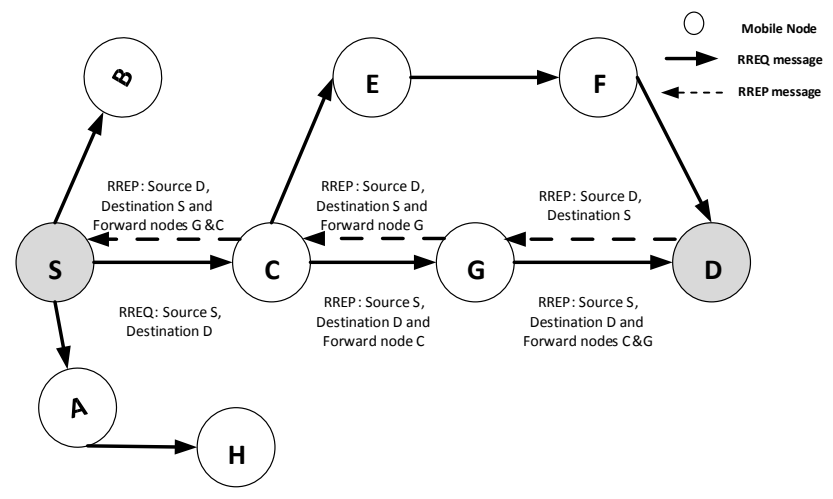

Fig. 2 - DYMO route discovery [15]

\subsection{Optimized Link State Routing}

OLSRv2 is an update and successor to OLSR as published in RFC3626. Currently, there is an active community around OLSRv2, and a proposed standard draft has been defined in RFC7181 by IETF MANET working group [16]. The OLSRv2 is developed for MANETs. Basically, it modifies the first version of OLSR by using and extending the following generalized building blocks: the MANET NeighborHood Discovery Protocol (NHDP), the Generalized MANET Packet/Message Format [RFC5444], the Type-Length-Values (TLVs) as specified in [RFC5497] and, optionally, Message Jitter as specified in [RFC5148]. According to [14], the OLSRv2 has been implemented using both a testbed built in Niigata University, Japan, and in the QualNet simulator [17]. An overview of OLSRv2 implementation is described in Figure 3 [18].

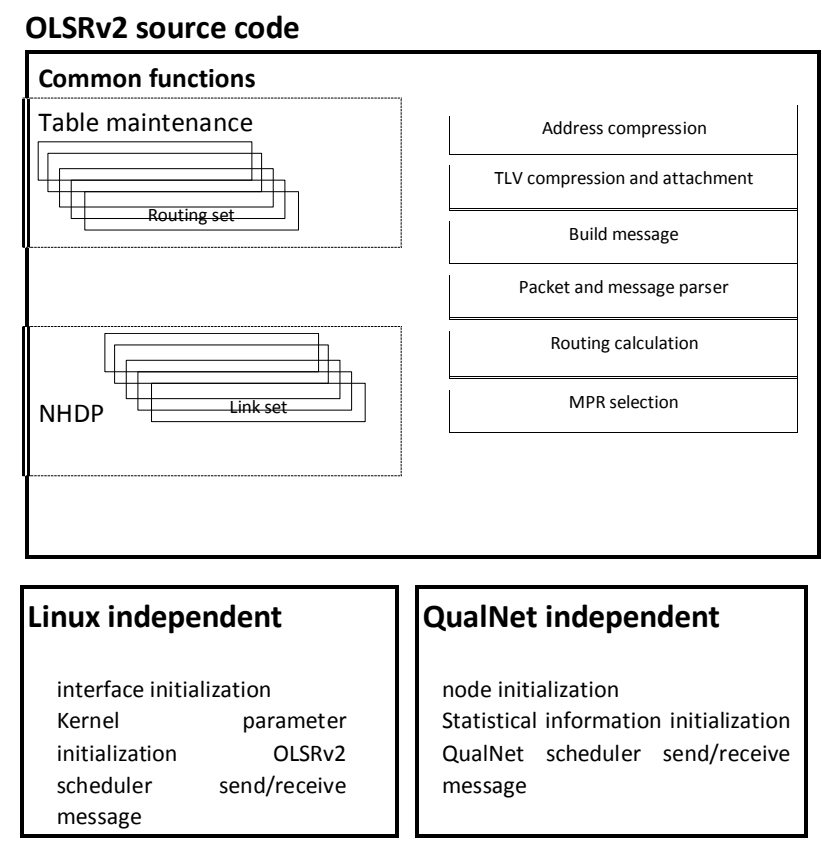

Fig. 3- OLSRv2 implementation overview redrawn from [14]

OLSRv2 is developed to operate independently from other protocols. The OLSRv2 makes no assumptions about the underlying link-layer, while it may use link-layer information and notifications when existing and being applicable. OLSRv2 [19] is in its final stage of standardization. It utilizes two basic types of control packets; HELLO Messages, and TC messages.

HELLO messages: In OLSRv2, HELLO messages have five functions: discovering links to adjacent OLSR nodes, performing bidirectional check on the discovered links, advertising neighbors and hence discover 2-hop neighbors, selecting single MPR, and advertising their own interfaces which participate in the MANET. The HELLO messages are emitted periodically, thereby, allowing nodes to continuously track changes in their local neighborhoods.

TC messages: In OLSRv2, TC messages serve to: inject link-state information into the entire network, inject addresses of hosts and networks for which they may serve as a gateway to the entire network, and allow nodes are with multiple interface addresses to ensure that nodes within two hops can associate these addresses with a single node for efficient MPR set determination. The TC messages are emitted periodically, thereby, allowing nodes to continuously track global changes in the network. 


\section{Related Work}

Many studies have been devoted to designing a routing protocol which is a crucial task, because of the highly dynamic topology that characterizes VANET. One of these studies was performed by the authors of [20] that carried out a comparison of four routing protocol, namely, DSDV, DSR, AODV and OLSR. The results of OLSR surpasses the other protocols in terms of throughput, end-to-end delay and PDR with high mobility conditions.

The study in [21] evaluated the performance of OLSRv2, DYMO, and MP-OLSR for three internet-based traffic applications. The evaluation was based on changing three main parameters in the simulation model namely, network size, number of traffic connections, and percentage of static nodes. The result shows significant benefits of MPOLSR which has the best performance over DYMO and OLSRv2 in terms of all investigated performance metrics for most scenarios in high density, heavy congestions, and high mobility environments as expected for all traffic types. DYMO has the best performance in terms of throughput, average end-to-end delay, average page request time, and consumed energy in the network with $100 \%$ static nodes compared with OLSRv2 and DYMO. OLSRv2 outperformed DYMO in terms of average time in queue in all simulations. There are different types protocols in VANET and can be classified according to their rout update method and position accusation as follows: Position Based Routing Protocol, Topology Based Routing Protocol, Broadcast Based Routing Protocol, Cluster Based Routing Protocol, and Geo Cast Based Routing Protocol.

AODV protocol's method to find the stable shortest connected path. The AODV performance improves the packet delivery ration in high densities. The AODV issues is when there is low traffic density, more packets cannot reach to the destination. OLSR protocol works based on active files. OLSR performance finds the shortest path with the minimum delay, and high packet delivery ratio as well reduced the waiting for vehicles at traffic jams [22]. Study done by [23] comparing the performance of AODV and OLSR protocols concludes, for small transmission rates, all packets sent from the source are received at the destination and the PDR is maximal for both protocols. For high transmission rate the PDR is decreased for both protocols but, the PDR of AODV is smaller than OLSR. For small transmission rates, the PDR of both protocols is maximal, and the throughput is theoretical. For big transmission rates, the OLSR has better throughput compared with AODV. The delay for small transmission rates is small for both protocols and they can be used for real time applications such as safety applications.

According to [24], the feasibility and the expected quality of VANETs operated with the routing protocol DYMO showed that for small amounts of payload data to be transported, ad hoc networks of vehicles and static highway infrastructure can be successfully setup, maintained, and used with well-known protocols from the Internet protocol suite alone. Even low node densities and sparse access point deployment sufficed to support routine polling of information via an Internet gateway. Larger amounts of network traffic to be transported over the ad hoc network, however, induced overload effects that noticeably destabilized the VANET. Particularly at higher node densities, which commonly occurred in micro-jams, the routing and transport protocol behavior led to a drastic increase in network load.

In [25] the study compared of four routing protocols DYMO, AODV, DSR and DSDV. The protocols were compared for low, medium and high traffic densities for a highway traffic scenario using 20, 40, and 60 nodes in the simulation on three performance metrics of End to End Delay, Packet Delivery Ratio and the Average Throughput. The result shows AODV has more PDR than DYMO and the difference in End to End delay is not too low than DYMO but when Throughput is concerned it is low than DYMO, DYMO on the other hand has low End to End delay and its PDR although less than AODV but the difference in PDR between the two continue to decrease as node density increases, its Throughput is high than AODV and continues to increase with the increase in the node density. Hence considering all these variations DYMO is the recommended protocol for a highway scenario.

\section{Methodology}

\subsection{Simulation Tools}

QualNet is a comprehensive suite of tools for modeling large wired and wireless networks. It uses simulation and emulation to predict the behavior and performance of networks to improve their design, operation and management. QualNet enables users to design new protocol models, optimize new and existing models, design large wired and wireless networks using pre-configured or user-designed models and analyze the performance of networks and perform what-if analysis to optimize them.

\subsection{Mobility Model}

The mobility model used in this paper is random way point. In this model the nodes move at random movements at different speed and no location restricted the nodes. For the VANET applications the random way point is used when the vehicle moves at different speed, pause time and other parameters that have varying characteristics, such as location, network size and so on. In the paper scenario the speed and the pause time of the vehicle changing over the time. So random way point model has selected for this scenario. 


\subsection{Simulation Environment}

All the simulation work is performed in QualNet wireless network simulator version 5.0. Initially number of nodes are 30, Simulation time was taken 100 seconds and seed as 1 . All the scenarios have been designed in $1500 \mathrm{~m} \times 1500 \mathrm{~m}$ area. Mobility model used is Random Way Point (RWP). In RWP a mobile node is initially placed in a random location in the simulation area. For simulation, environmental surrounding selected is Pause time. Pause time is varying between the ranges of 30-110 sec. "Pause time is a time in which all nodes in network are motionless but transmission in continued". And node speed of range 3-12 m. All the simulation works were carried out using routing protocol DYMO and OLSRv2 with varying pause time and node speed. Network traffic load is provided by constant bit rate (CBR) application [26]. A CBR traffic source provides a constant stream of packets throughout the whole simulation, thus further stressing the routing task.

\subsection{Performance metrics}

The meaning of metric refers to the numeric value used to describe the quality of a particular route as good or bad. However, the performance metrics refers to the numeric values used to test the performance of routing protocols. The performance of routing protocols can be evaluated with respect to some metrics. The most important metrics used to evaluate routing protocols are Packet Delivery Ratio (PDR) that is the ratio of the total packets received at the destination to the number of packets generated by the same node.

Another performance metrics is the Average Jitter rate is the time delay between consecutive packets that reach the same destination. End-to-end Delay is the average time taken for the packets to travel from the end source to the end destination, delay of the data is very important to be considered because data going through the network may take longer time to reach destination. Throughput is to measure the successful packets deliver to the destination over period or simulation time.

Table 1 - Simulation Parameters Setting

\begin{tabular}{ll}
\hline \multicolumn{1}{c}{ Parameter } & \multicolumn{1}{c}{ Value } \\
\hline Simulator & QualNet Version 5 \\
Terrain Size & $1500 \times 1500 \mathrm{~m} 2$ \\
Antenna model & Omni-directional \\
No of nodes & 30 \\
Radio Type & $802.11 \mathrm{~b}$ \\
Data size & $512 \mathrm{bytes}$ \\
Data Rate & $2 \mathrm{Mbps}$ \\
Mobility Model & Random Way Point \\
Channel Frequency & $2.4 \mathrm{GHz}$ \\
Traffic Source & Constant Bit Rate \\
Item to send & 100 \\
Routing Protocols & DYMO \& OLSRv2 \\
Traffic type & CBR \\
Simulation time & $100 \mathrm{~s}$ \\
Start time & $10 \mathrm{~s}$ \\
End time & $0 \mathrm{~s}$ \\
Pause time & $30 \mathrm{~s}, 50 \mathrm{~s}, 70 \mathrm{~s}, 90 \mathrm{~s}, 110 \mathrm{~s}$ \\
Nodes speed & $3 \mathrm{~m} / \mathrm{s}, 7 \mathrm{~m} / \mathrm{s}, 12 \mathrm{~m} / \mathrm{s}$ \\
\end{tabular}

\section{Results and Discussions}

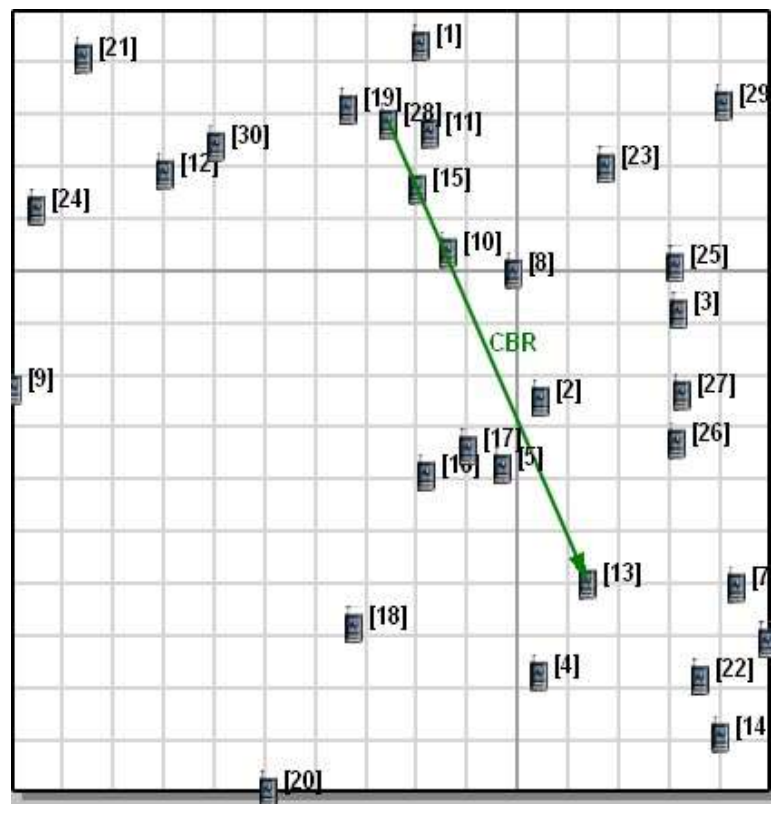

Fig. 4 - Simulation scenario

The system evaluation will be based on comparing two protocols DYMO and OLSRv2. Varying node speed is used at three different speeds of vehicle such as 3, 7 and 12 meters. Another parameter used is pause time at five different pause time such as 30, 50, 70, 90 and 110 second. When the speed of the node changes from time to time the performance of the system also changing. Similar to the pause time when change the pause time the performance of the system as well changing. There are four criteria for system evaluation to know how much the changing in the system, and they are as follows: average jitter, throughput, end-to-end delay and packet delivery ratio.

\subsection{The Impact of Varying Pause Time}

In the system there are stopping times from time to time either to maintenance of the system or to run other applications and stop other for a while. In VANET as well the node can be in pause time state in traffic jam or at traffic light as well maintenance and so on. Based on the protocol mechanism how the protocol dealing with the pause time. Every protocol has its own characteristics that differentiate it from other protocols. Figure 5 represents the effects of the 
average jitter over the changing of pause time. As the graph shows that the OLSRv2 routing protocol has better average jitter in all the pause time changing compare to DYMO routing protocol. In OLSRv2 the packets reach the destination with small jitter time compare to DYMO protocol. OLSRv2 routing protocol has higher performance in average jitter compare to DYMO routing protocol.

Packet delivery ratio is calculated by how many total packets received over total packets send. In the packet delivery ratio, the DYMO routing protocol has higher performance compare to the OLSRv2. Figure 6 shows the different between the DYMO and OLSRv2 in packet delivery ratio is significant. If the system wants high packet delivery ratio, it should select DYMO routing protocol over the OLSRv2routing protocols. End-to-end Delay: the average time taken by a data packet to arrive in the destination. It also includes the delay caused by route discovery process and the queue in data packet transmission. Only the data packets that successfully delivered to destinations that counted. In Figure 7, at the Average End-to-End Delay the system performance evaluation it shows that, the OLSRv2 has much better performance compare to DYMO protocol. The delay time between the packet sent from the end source to the receiver node end is less in OLSRv2 routing protocol.

The system throughput is to calculate the packet received over period. Figure 8 represents the different in the system performance of the two protocols (DYMO and OLSRv2). Figure 8 shows that the DYMO protocol has much higher value compare to OLSRv2 protocol. The system has higher performance base on the paper scenario with the DYMO protocol. OLSRv2 has low performance of throughput in the system. DYMO protocols should be selected if the system required high throughput.

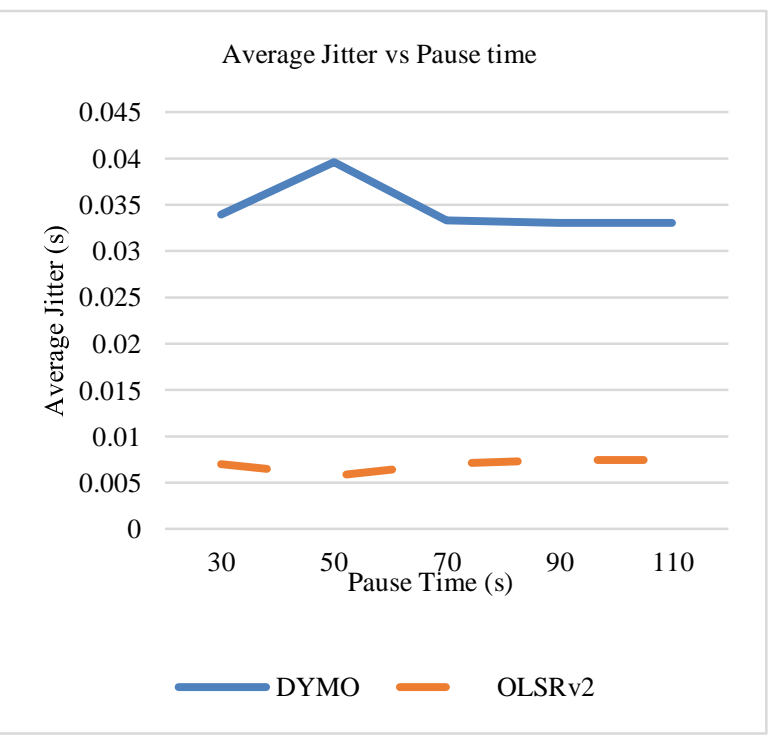

Fig. 5- Average Jitter vs. Pause time

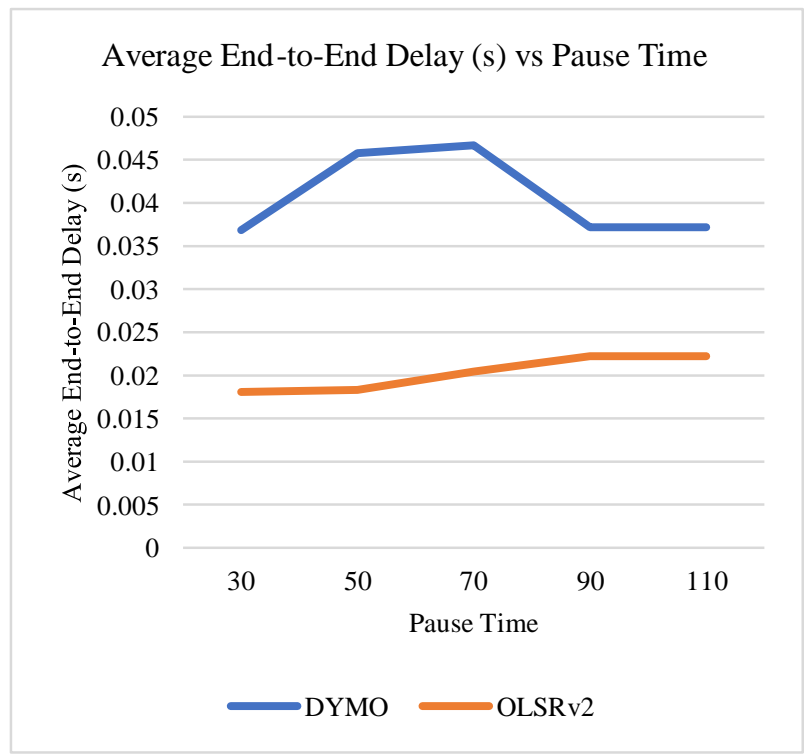

Fig. 7 - End-to-End delay vs. Pause time

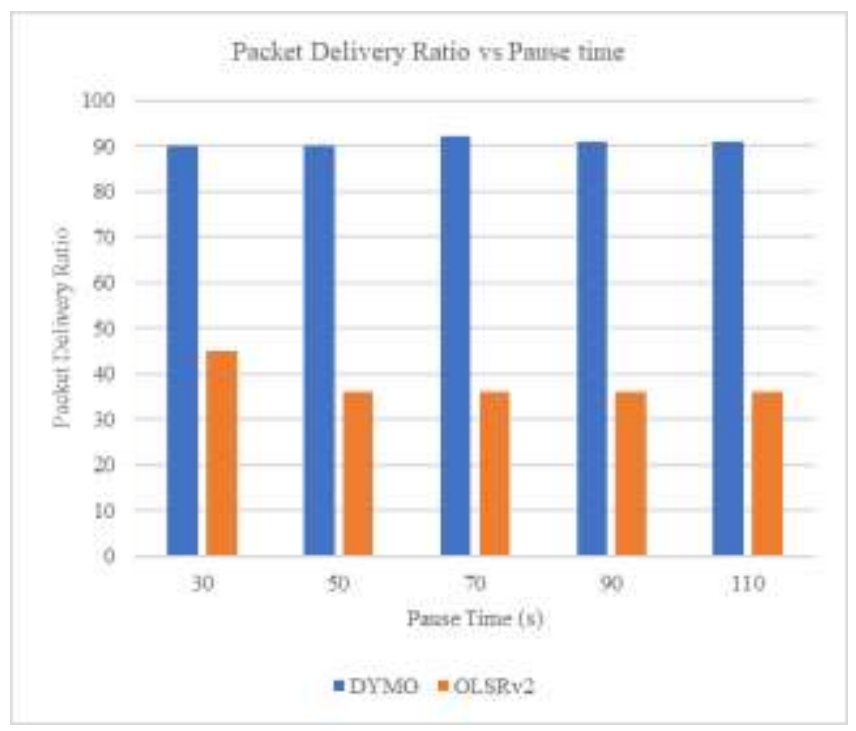

Fig. 6 - Packet delivery ratio vs. Pause time

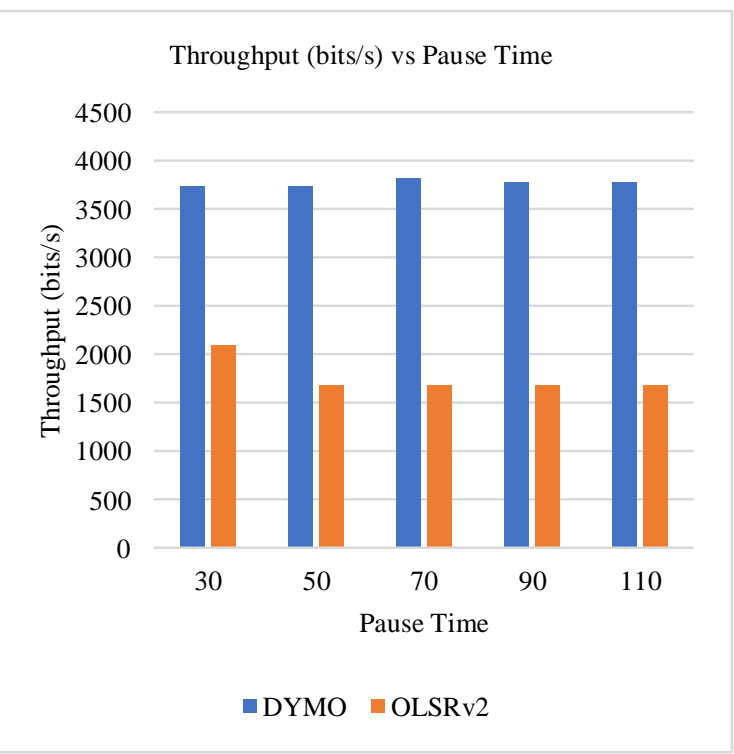

Fig. 8 - Throughput vs. Pause time 


\subsection{The Impact of Varying Node Speed}

The node speed has a great effect on the system performance. In VANET network has the capability to support high speed such speed in highway may reach over $160 \mathrm{~km} / \mathrm{h}$. Some of the node does not support high speed due to the other factors that may result in packets dropping, such as, packet size, range, time and so on. In this simulation result, the output will explore the effect of the speed of node changing on the system performance. The system will test on three different speeds, at (3, 7 and 12) meters. The system will be evaluated based on the four criteria, average jitter, throughput, endto-end delay and packet delivery ratio. Based on the result of these criteria, the protocol will be selected for the specific solution.

Figure 9 represents both protocols have different result for the same scenario. OLSRv2 shows high performance in all speed changing compare to DYMO protocol. OLSRv2 has high performance comparing to DYMO protocol. If the system desire low average jitter, the system should select OLSRv2 protocol over DYMO. The output of the PDR shows in Figure 10 that, the DYMO protocol has better performance compare to OLSRv2. The ratio of packet delivery at the DYMO protocol much higher than OLSRv2. The difference in the output for the two protocols are significant. DYMO protocol has high PDR in the system because its support high data transmission even if the node moving in high speed like in VANET highway. If the system required high packet delivery ration, it should select DYMO protocol over OLSRv2 protocol.

OLSRv2 shows great response to the average end-to-end Delay compare to the DYMO protocol. At all three different speed, the OLSRv2 show low delay in packet end-to-end delivery. Figure 11 shows how the output changed when the speed of the node increased. OLSRv2 protocol has better performance in AEED due to the proactive routing protocol which allows the protocol to select the best path from the routing table that already prepared when there is a request of establishing path and send the data immediately to destination. Figure 12 shows DYMO protocol has higher throughput because of it can send high data over slot of time, that allows the system to get high throughput in DYMO protocol. The system has much higher performance with DYMO protocol over OLSRv2 protocol. If the system desire to get high throughput, the system should select DYMO protocol.

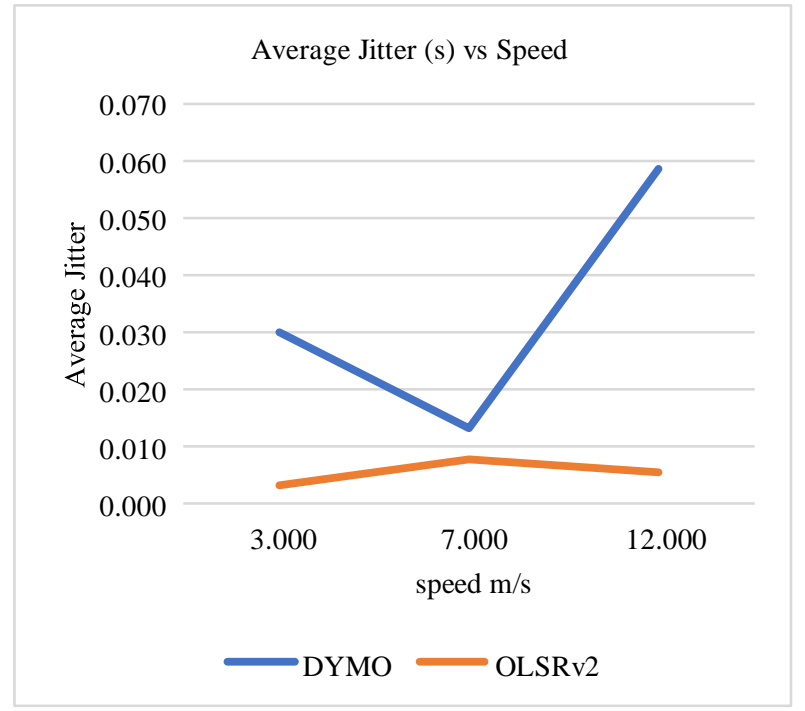

Fig. 9 - Average Jitter vs. Speed

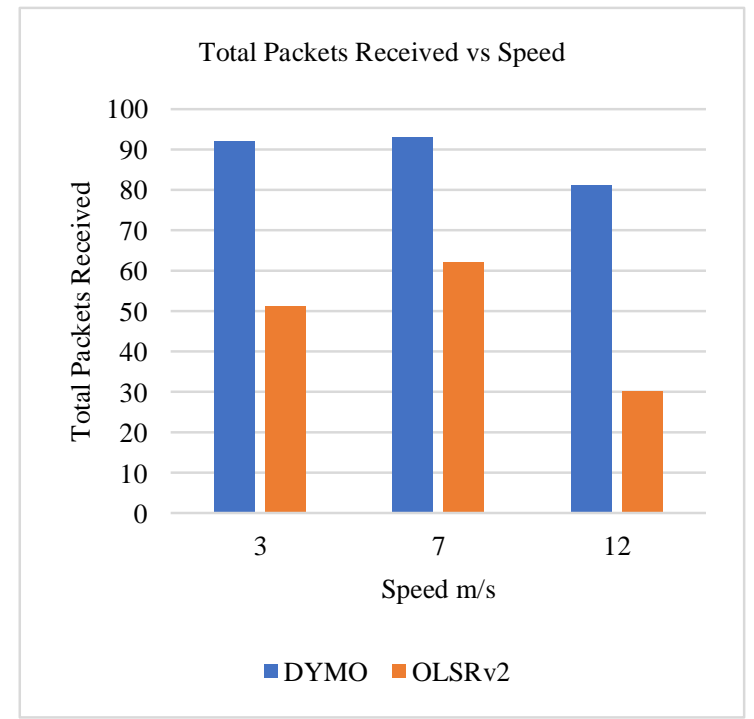

Fig. 10 - PDR vs. Speed 


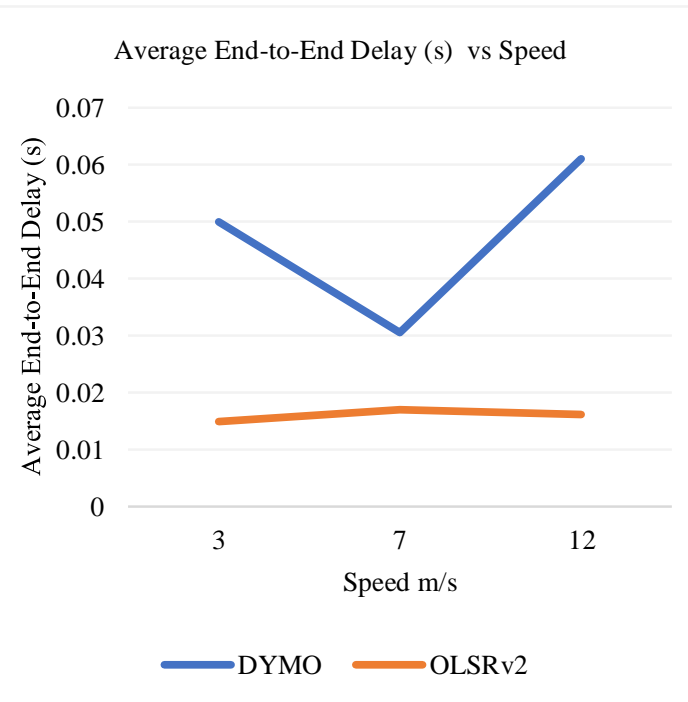

Fig. 11 - End-to-End delay vs. Speed

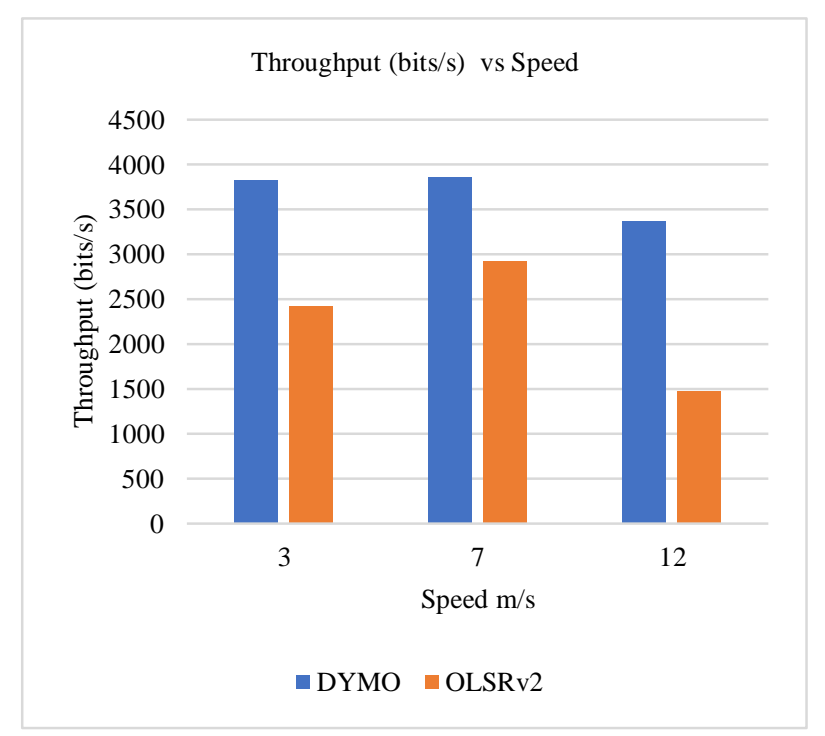

Fig. 12 - Throughput vs. Speed

\section{Conclusion}

This paper has evaluated two types of different routing protocols such as DYMO and OLSRv2 routing protocols. The output of the simulation shows that in some of criteria such as packet delivery ratio and throughput, DYMO protocol is optimal choice to be selected compare to OLSRv2 protocol. However, this preference does not continue with other criteria such as average jitter and end-to-end delay, the result shows that, the OLSRv2 protocol is optimal for these criteria compare to DYMO protocol. The system designer should select DYMO protocol over OLSRv2 if the targeting is to achieve high performance in throughput and packet delivery ratio. However, if the system designer targeting high performance in average jitter and end-to-end delay, OLSRv2 protocol should be selected over DYMO protocol.

As a future work, these two protocols can be improved in the term of security such as the system should only allow the node that authorized to be participated, and other types of security if third party wants to connect to the network. The system can be improved in connection for smart city for $5 \mathrm{G}$ connection. The system should be improved in the data link layer to be able to merge with the future technologies and other systems. The system can be improving in the way of supporting business, which allow the companies to provide the services to the users and charge them for it.

\section{Acknowledgment}

This research was supported in part by Atatürk Üniversitesi Kampüsü, Turkey and in part by Universiti Malaysia Pahang (www.ump.edu.my), Malaysia under the Grant Scheme UMP- IBM Centre of Excellence No. RDU190304.

\section{References}

[1] M. Conti and S. Giordano, "Multihop Ad Hoc Networking: The Evolutionary Path," Mobile Ad Hoc Networking: The Cutting Edge Directions, vol. 35, p. 3, 2013.

[2] W. A. Jabbar, M. Ismail, and R. Nordin, "MBA-OLSR: a multipath battery aware routing protocol for MANETs," in Intelligent Systems, Modelling and Simulation (ISMS), 2014 5th International Conference on, 2014, pp. 630635.

[3] W. A. Jabbar, M. Ismail, R. Nordin, and R. M. Ramli, "EMA-MPR: Energy and mobility-aware multi-point relay selection mechanism for multipath OLSRv2," in Communications (MICC), 2017 IEEE 13th Malaysia International Conference on, 2017, pp. 1-6.

[4] M. Conti and S. Giordano, "Multihop ad hoc networking: the evolutionary path," Mobile Ad Hoc Networking: Cutting Edge Directions, pp. 1-33, 2013.

[5] Almohammedi, A. A., Noordin, N. K., Sali, A., Hashim, F., \& Balfaqih, M. (2018). An Adaptive Multi-Channel Assignment and Coordination Scheme for IEEE 802.11 P/1609.4 in Vehicular Ad-Hoc Networks. IEEE Access, 6, 2781-2802.

[6] B. T. Sharef, R. A. Alsaqour, and M. Ismail, "Vehicular communication ad hoc routing protocols: A survey," Journal of network and computer applications, vol. 40, pp. 363-396, 2014.

[7] S. Khan, M. Alam, M. Fränzle, N. Müllner, and Y. Chen, "A Traffic Aware Segment-based Routing protocol for VANETs in urban scenarios," Computers \& Electrical Engineering, vol. 68, pp. 447-462, 2018.

[8] W. A. Jabbar, M. Ismail, and R. Nordin, "Energy and mobility conscious multipath routing scheme for route stability and load balancing in MANETs," Simulation Modelling Practice and Theory, vol. 77, pp. 245-271, 2017.

[9] T. Clausen, C. Dearlove, and P. Jacquet, "The optimized link state routing protocol version 2," draft-ietfmanetolsrv2-00, Work in progress, 2006. 
[10] U. Herberg, T. Clausen, P. Jacquet, and C. Dearlove, "The Optimized Link State Routing Protocol Version 2 ," 2014.

[11] I. Chakeres and C. Perkins, "Dynamic MANET on-demand (DYMO) routing," draft-ietf-manet-dymo-14 (work in progress), 2008.

[12] I. Chakeres and C. Perkins, "Dynamic MANET On-demand (AODVv2) Routing draft-ietf-manet-dymo-26," IETF Internet-Draft, http://tools. ietf. org/id/draft-ietf-manet-dymo-26. txt2014.

[13] J. Dowdell, S. Ratliff, and C. E. Perkins, "Dynamic MANET On-demand (AODVv2) Routing," 2013.

[14] N. Sivakumar and S. K. Jaiswal, "Comparison of DYMO protocol with respect to various quantitative performance metrics," Department of Computer Science, Malardalen University, 2009.

[15] A. K. Gupta, H. Sadawarti, and A. K. Verma, "Implementation of DYMO Routing Protocol," arXiv preprint arXiv:1306.1338, 2013.

[16] J. Yi, "Network Working Group T. Clausen Internet-Draft Intended status: Informational U. Herberg Expires: February 13, 2015 Fujitsu Laboratories of America," 2014.

[17] P. Jacquet and U. Herberg, "Internet Engineering Task Force (IETF) T. Clausen Request for Comments: 7181 LIX, Ecole Polytechnique Category: Standards Track C. Dearlove," 2014.

[18] N. S. Qualnet "Scalable Network Technologies," Inc.[Online]. Available: www. qualnet. com, 2011.

[19] Y. Owada, K. Tsuchida, T. Maeno, H. Imaix, and K. Mase, "OLSRv2 Implementation and Performnce Evaluation," in 3rd OLSR Workshop, 2006.

[20] Mohapatra, S., \& Kanungo, P. (2012). Performance analysis of AODV, DSR, OLSR and DSDV routing protocols using NS2 Simulator. Procedia Engineering, 30, 69-76.

[21] W. A. Jabbar, M. Ismail, R. Nordin, and R. M. Ramli, "Traffic load-based analysis of MBQA-OLSR routing protocol in wireless ad hoc networks," in Region 10 Conference, TENCON 2017-2017 IEEE, 2017, pp. 26772682.

[22] Brendha, R., \& Prakash, V. S. J. (2017, January). A survey on routing protocols for vehicular Ad Hoc networks. In Advanced Computing and Communication Systems (ICACCS), 2017 4th International Conference on (pp. 17). IEEE.

[23] Spaho, E., Ikeda, M., Barolli, L., Xhafa, F., Younas, M., \& Takizawa, M. (2013, March). Performance evaluation of OLSR and AODV protocols in a VANET crossroad scenario. In Advanced Information Networking and Applications (AINA), 2013 IEEE 27th International Conference on (pp. 577-582). IEEE.

[24] Sommer, C., \& Dressler, F. (2007, September). The DYMO routing protocol in VANET scenarios. In Vehicular Technology Conference, 2007. VTC-2007 Fall. 2007 IEEE 66th (pp. 16-20). IEEE.

[25] Thakur, A. S., \& Ganpati, A. (2013). A Comparative Study of DYMO AODV DSR \& DSDV Routing Protocols in VANET. International Journal of Advanced Research in Computer Science and Software Engineering, 3(10). 